\title{
DISCUSSION
}

\section{Change in pore size distribution due to consolidation of clays}

\author{
F. J. GRIFFITHS and R. C. JOSHI (1989). Géotechnique, 39, No. 1, 159-167
}

T. S. Nagaraj, A. Vatsala and B. R. Srinivasa Murthy, Indian Institute of Science, Bangalore

The Paper by Griffiths and Joshi is an important step in understanding the process of consolidation from microstructural considerations. The Authors have made a meticulous experimental investigation. However, a little deeper observation indicates that the results can be interpreted to reveal several microstructural changes during compression and also the limitations of mercury porosimetry in providing the information about particle clustering at submicroscopic level.

The generalized compression equation

$$
e / e_{\mathrm{L}}=1 \cdot 122-0.2343 \log p
$$

quoted in the Paper (equation (3)) takes into account the type of soil in terms of void ratio, at liquid limit $e_{\mathrm{L}}$. The above relationship has been based on Gouy-Chapman diffuse double layer theory (Nagaraj \& Srinivasa Murthy, 1986). The physical model was built for fine grained soils, for clay particles in an ideal situation of parallel configuration. It has been shown earlier that there is a unique relation between the interparticle distance, $2 d$ and the net repulsive pressure $(R-A)$ mobilized, which is in equilibrium with the externally applied pressure $P^{\prime}$ (Sridharan \& Jayadeva, 1982; Nagaraj \& Srinivasa Murthy, 1986). The micro to macrolevel link was drawn from the relation $e=G \gamma_{\mathrm{w}} S d$ for such an ideal parallel plate condition. It is, however, known and can also be seen from the results of porosimetry tests that in reality, at a given pressure, the soil has pores of a wide range of sizes (and not a single value of $d$ ). At the same time it is also true that in fine grained soils the mode of stress transfer is through physico-chemical interactions and in some way is related to the double layer theory. Hence the test results are re-examined to explain the microstructure with this background.

The double layer repulsion $R$ and the van der Waals attraction $A$ (and hence the net interaction force between two charged particles) are influenced by the properties of pore fluid and environment. Except for very high electrolyte concentrations, the net interaction energy will be as shown in Fig. 9. (van Olphen, 1963) exhibiting a minimum or predominant attraction (point $\mathrm{A}$ ) at close spacing, and a maximum with predominant repulsion at greater distances. For the usual field environment in clays, computations of $R$ using the double layer equation and of $A$ using Hamaker's equation have shown (Sridharan \& Jayadeva, 1982; Nagaraj \& Srinivasa Murthy, 1986) that there is practically no force exerted beyond a distance of about $200-300 \AA$ (point $D$ in Fig. 10). The distance at which the particles cross the repelling barrier may be about $20 \AA$ (point C) and hence net energy may be one of attration at much closer spacing (point $B$ ). Hence if the particles come closer than $20 \AA$, they get attracted to form clusters or aggregates which act as a single unit. Then the externally applied pressure is to be transferred across two such units or aggregates and the distance between them will be governed

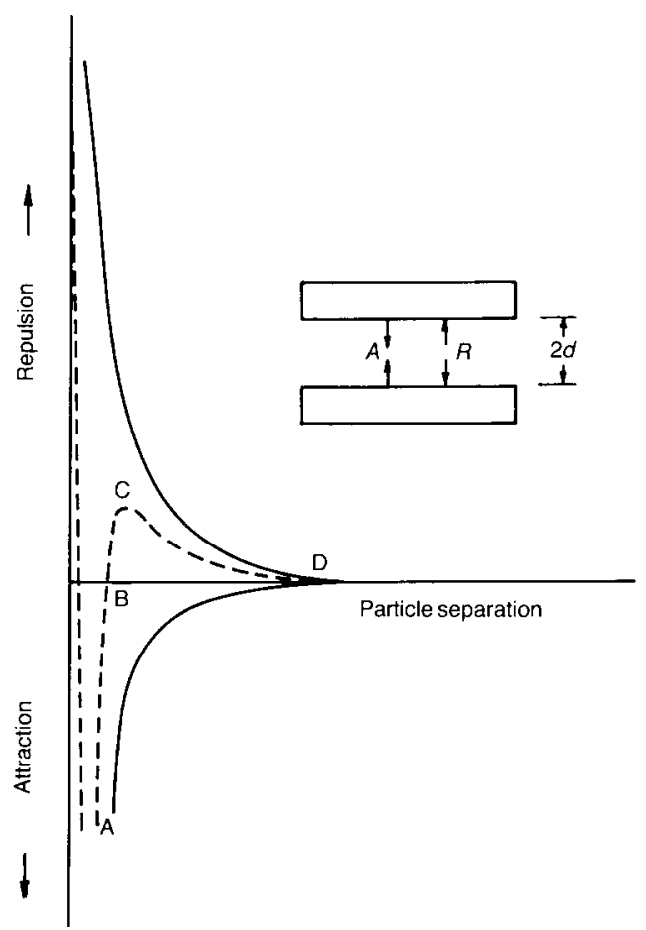

Fig. 9. Net interaction energy diagram for intermediate electrolyte (after Van Olphen, 1963) 


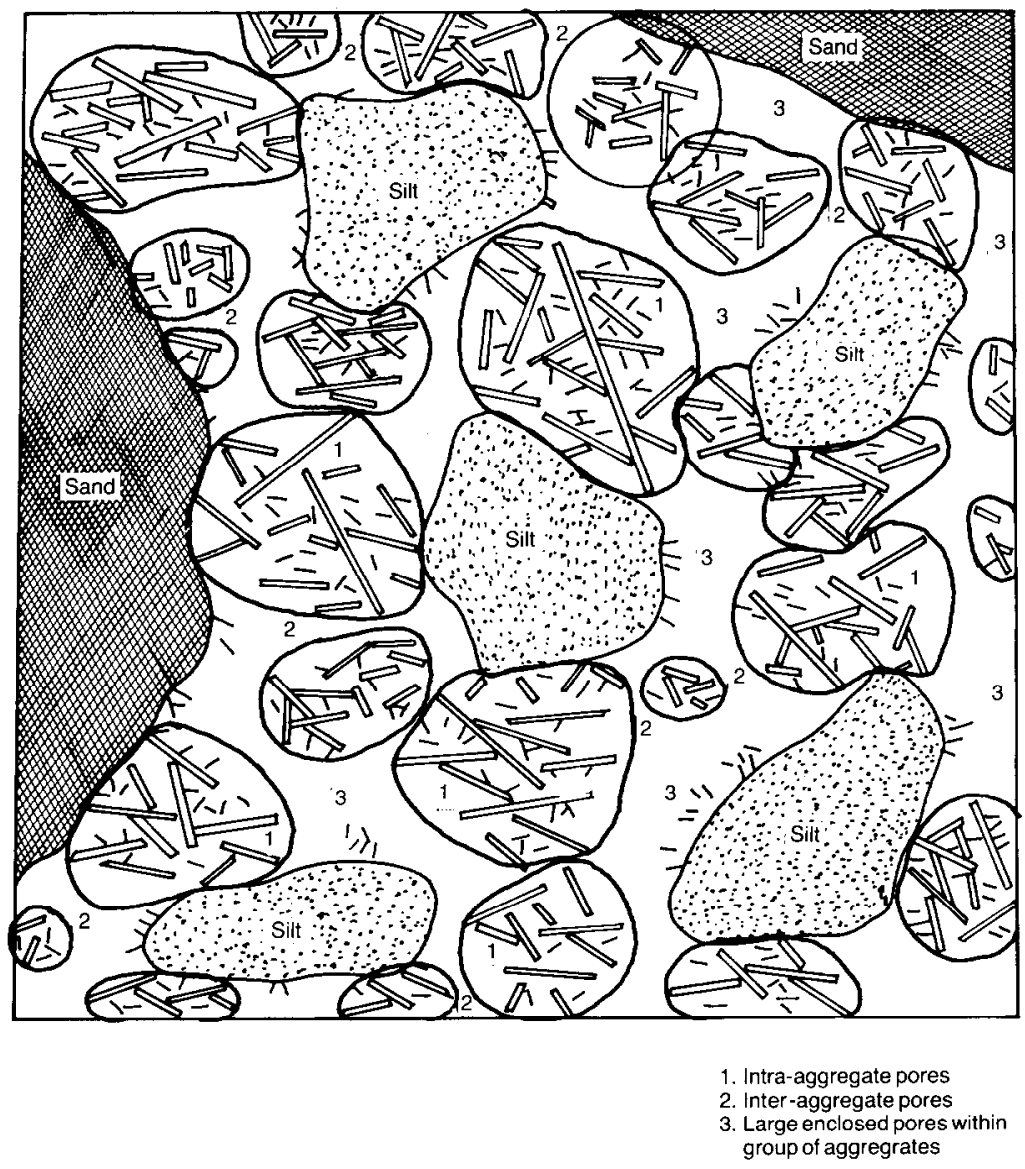

Fig. 10. Microstructure of fine grained soil

by double layer considerations to mobilize the required repulsive force. But it can be seen from the pore size distribution curves of Figs 2-6 that in all the four soils there are pores of sizes much larger than $300 \AA$, and that the volume of such pores is more than about $90 \%$ of the total volume of pores (or the volume of pores $<200 \AA \mathrm{dia}$. is not more than $5-10 \%$ of the total volume of pores). This means $90 \%$ of the pore volume is not directly participating in clay-surface interactions. This is supported by water content calculations also. For example, if it is assumed the thickness of a kaolinite particle as $650 \AA$ (usually in the range $300-1000 \AA$ ), for a maximum thickness of water film of $200 \AA$ at liquid limit, its water content can be calculated as

$$
\begin{aligned}
W & =\frac{\text { weight of water }}{\text { weight of solids }} \\
& =\frac{L \times B \times 200 \AA \times 1}{L \times B \times 650 \AA \times 2.7}=11.3 \text { or } \simeq 12 \%
\end{aligned}
$$

(assuming specific gravity of solids as $2 \cdot 7$ ), whereas the actual liquid limit water content for pure kaolinite will be much more than this $(\simeq 80 \%)$. This implies that all the water present even at liquid limit is not participating in double layer interactions.

It appears that the existence of three levels of pores (Fig. 10) can be identified

(a) Intra-aggregate pores between the individual platelets in an aggregate with pore diameter $<20 \AA$

(b) inter-aggregate pores between two interacting aggregates with diameter $<200 \AA$ and $>20 \AA$ depending on the applied pressure

(c) intra-aggregate large pores held within a group of clusters, with diameter $\gg 200 \AA$.

The Authors' results indicate that it is only the large size pores which are affected by consolidation pressure, although these pores are not directly influenced by clay-surface interactions. This can be explained as follows. The large pores 
have to be in equilibrium with the surrounding force system and the size of such pores enclosed within the group of clusters is also controlled by the same force system and it cannot be arbitrary. This is the reason for obtaining the generalized form of the equation by the Authors. Thus the sizes of both inter-aggregate (type 2) and large enclosed (type 3) pores are dictated by the applied force system. It may be that the ideal configuration of pores can be visualized at any given equilibrium state as having three different but definite sizes of pores as shown in Fig. 11. $R_{1}$ corresponds to intra-aggregate pores, $R_{2}$ to interaggregate pores and $\boldsymbol{R}_{3}$ to the large enclosed pores. However, in a random system with soil particles of different sizes and stressed differently in three different directions the actual curve will be a smooth one covering these pore ranges. There is one other additional reason for the observed distribution of pore sizes. The porosimetry records sizes of pore entrance and not of the enclosed pores. It is possible in a continuous system that a pore of a given radius has different sizes of connecting paths or entries of flow with the surrounding system.

With increase in stress, the distance between two interacting units will reduce and the size of the large enclosed pores will have to reduce. To accommodate this reduction in size, it may be necessary for the aggregates to reform or to group together to form larger clusters. Such grouping will have to result in small changes in the volume of pores of sizes $<20 \AA$ and also of size $2 d$ but the volume of such pores is only about $5-10 \%$ of the total volume, such small changes will not be noticeable or detectable from mercury intrusion tests, $(5 \%$ of 5 , i.e. about 0.25 of the total pore volume). As the results show, the first intrusion and second intrusion curves are practically the same within this range of pore sizes. Mercury porosimetry tests therefore are not the right diagnostic tests to prove or to rule out the possibility of formation of larger clusters with increase in stress. The research work at the Indian Institute of Science has indicated that stress-strain behaviour of fine grained soils can be predicted well using the simplest Cam-clay or other plasticity models if we postulate a gradual formation of larger clusters with increase in stress, and their dismembering during shearing.

The pore size distribution curves at liquid limit (which correspond to a definite equilibrium state of about $6 \mathrm{kPa}$ of consolidation pressure) in Fig. 2 confirm the above thoughts. The size of the largest pores is very nearly of the same order for all the four soils even though their liquid limit water contents vary from 29 to $100 \%$-i.e. there may be a greater number of such pores in soils with higher liquid limits although the size of pores is the same in all the soils.

With increase in pressure the size as well as total volume of the large enclosed pores should also decrease correspondingly. The test results of each of the soils indicate such a tendency qualitatively, but the reported results may not be giving the true picture because the tests have been conducted on samples after releasing the consolidation pressure and allowing the soil to rebound, i.e. the state of the soil no longer corresponds to the applied higher stress level but instead corresponds to the zero stress level (in an overconsolidated state). A more realistic picture of the pore size distribution at different stresses can be obtained by taking samples for porosimetry tests without allowing the soil to take in water on stress release.

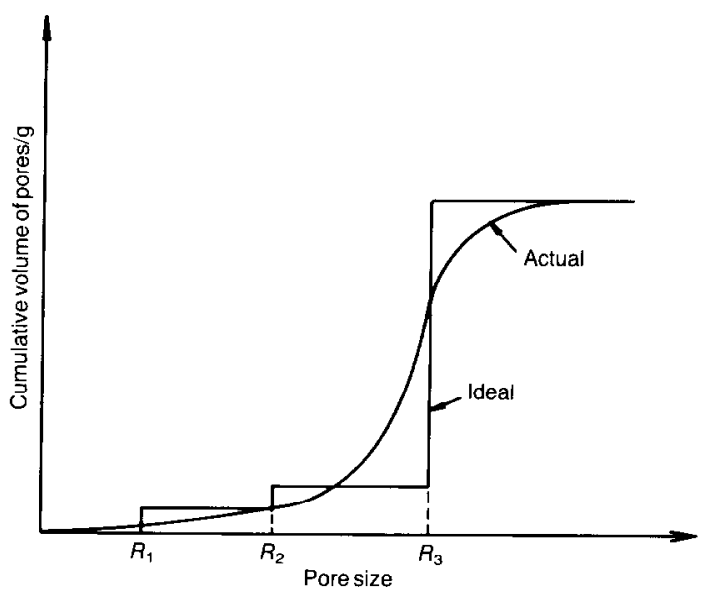

Fig. 11. Pore size distribution 
Table 2. Comparison of water contents and total introduced mercury volume

\begin{tabular}{l|c|c}
\hline & $\begin{array}{c}\text { Water filled } \\
\text { porous volume: } \\
\mathrm{ml} / \mathrm{g}\end{array}$ & $\begin{array}{c}\text { Total intruded } \\
\text { mercury volume: } \\
\mathrm{ml} / \mathrm{g}\end{array}$ \\
\hline 1 & 0.29 & 0.31 \\
2 & 0.45 & 0.52 \\
3 & 0.65 & 0.70 \\
4 & 1.00 & 1.04 \\
\hline
\end{tabular}

Study of the relationship between microstructure and the macroscopic behaviour of fine grained soils is difficult, and the Paper gives useful results in that direction. Because consolidation involves expulsion of water from collapsed pores, MIP has been revealed to be a particularly well adapted investigation technique.

The Paper's results, indicate a very good correlation between the soil water contents and the total intruded mercury volume, at $30 \mathrm{kPa}$ for instance (Table 2). This means that dehydration occurred with little volume change and that mercury fills all the voids, which were previously full of water. Pores with radii smaller than $2 \mu \mathrm{m}$ should therefore not exist.

The deformable aggregates type of model for consolidation appears to be quite general, as it seems to apply to compacted, natural, sensitive soft soils and different reconstituted soils. In all those cases, the application of macroscopic stresses in the $01500 \mathrm{kPa}$ range is able to modify only those pores whose radius is no smaller than $0.1 \mu \mathrm{m}$. Such a distance seems bigger than the range of action involved according to the double layer theory, which is in the order of several tens of nanometers. Such models should therefore be used cautiously in the interpretation of the consolidation process of soft soils. It has been observed that capillary actions of drying involve much stronger microscopic stresses and are able to affect smaller pores.

In the case of consolidated samples it is necessary to unload before running MIP, which includes some swelling. SEM observations on sensitive clays showed some sub-horizontal fissures which were believed to correspond to this swelling. Such a porosity is measured by the first intrusion curve and is believed to be an entrapped one, not measured by the second intrusion. The relative part of this porosity may be estimated from consolidometer curves. It is more important for higher stresses, particularly for the two looser samples ( 3 and 4).

The Authors have presented a relation between inter-aggregate porosity and consolidation stress. It is also suggested that correlation of the slope $C_{\mathrm{p}}$ of the pore size distribution (PSD) curve with
Table 3. Comparison between compression index $C_{\mathrm{c}}$ and slope $C_{\mathrm{p}}$ of PSD curve

\begin{tabular}{c|c|c}
\hline & $C_{\mathrm{c}}$ & $C_{\mathrm{p}}$ \\
\hline 1 & 0.191 & 0.470 \\
2 & 0.154 & 0.145 \\
3 & 0.466 & 0.421 \\
4 & 1.27 & 1.09 \\
\hline
\end{tabular}

the compression index of the clay should be made. In this case, it is necessary to plot the PSD curve using the intruded volume/grain volume ratio, which is the equivalent of the void ratio. Such a comparison is presented for the Paper's data in Table 3. Except for soil 1, the correspondence is not too bad. (For soil 2, the smaller slope, which corresponds to the poorly-graded bigger pores family, has been considered).

The same correspondence has becn observed for various Canadian sensitive clays (Delage, 1987) and is presented in Table 4. In this case, as Fig. 12, shows no shift towards smaller pores is observed between two stress values. It is concluded that a given stress increase collapses only the larger existing pores and has no effect on the smaller ones, which, due to their size, are strong enough to resist. This behaviour is related to the brittleness of the solid matrix of those cemented clays, which allows a clear segregation of the pores according to their size, and to the maximum stress they are able to resist. Then a relation may be established between a given stress and the size of the pores that this stress is able to collapse. In such conditions, the parallel between MIP and consolidometer curves is clear: in the first case, a given level of mercury pressure intrudes a pore family whose mean radius is defined by the capillary law; in the compression process, a given stress increase collapses only pores of a given size, whose porous volume is measured by the void ratio decrease. A compressible soil (high $C_{\mathrm{c}}$ ) will therefore have a better graded pore distribution (high $C_{\mathrm{p}}$ ).

Table 4. Comparison between compression index $C_{\mathrm{c}}$ and slope $C_{\mathrm{p}}$ of PSD curve, for various sensitive Canadian clays (Delage, 1987)

\begin{tabular}{l|l|c}
\hline & $C_{\mathrm{c}}$ & $C_{\mathrm{p}}$ \\
\hline St Marcel & $2 \cdot 28$ & $2 \cdot 42$ \\
St Guillaume & $2 \cdot 27$ & $2 \cdot 53$ \\
St Louis & $2 \cdot 23$ & $2 \cdot 48$ \\
St Alban & $1 \cdot 4$ & 1.43 \\
St Thuribe $(3,40 \mathrm{~m})$ & $0 \cdot 70$ & $0 \cdot 80$ \\
St Thuribe $(5,77 \mathrm{~m})$ & $1 \cdot 07$ & $0 \cdot 85$ \\
St Léon & $2 \cdot 6$ & 2.79 \\
Broadback B2 & $1 \cdot 4$ & 1.74 \\
\hline
\end{tabular}




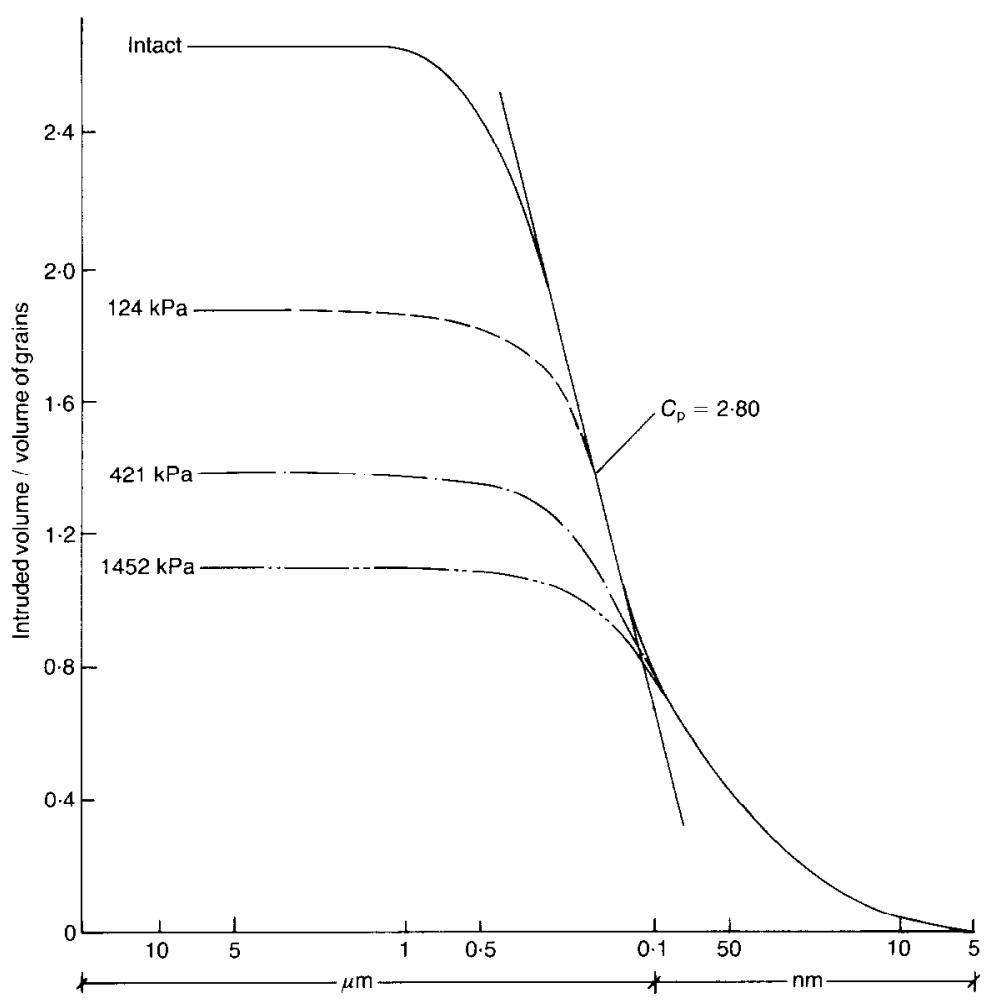

Fig. 12. Change in pore size distribution due to consolidation of St Marcel clay (Delage, 1987)

As the brittleness of the solid matrix of the reconstituted samples studied in the Paper is much less, the parallel may be less evident. However, the $C_{\mathrm{p}} / C_{\mathrm{c}}$ correspondence is reasonable.

A further approach would be to look out for some correlations between mineralogy and poresize distribution, assisted by SEM observations and by data on of the relative proportions of the different minerals. The two softer samples $(3$ and 4), unlike soil 1, should have an amount of montmorillonite sufficient to correspond to a higher liquid limit and total initial pore volume. As described by the Authors, the evolution of the MIP curve of those two soils during consolidation is similar, with a slight shift towards smaller porosity with increasing stress. Soil 1 exhibits a similar trend, but seems to be more sensitive to the $1500 \mathrm{kPa}$, where smaller pores are formed.

The pure kaolinite soil (2) has a characteristic PSD curve, with a poorly graded distribution of bigger pores $(>0.15 \mu \mathrm{m})$, and a well graded family of small pores $(<0.15 \mu \mathrm{m})$, which corresponds to $60 \%$ of the total porosity at the liquid limit. Those smaller pores are not affected by the consolidation, and their distribution remains remarkably invariant. Such a PSD curve should probably correspond to a well crystallized kaolinite structure, which has been described by Tovey (1971) in Mitchell (1976) (p. 36). In this case, welldefined stacks of $0.2 \mu \mathrm{m}$ thick plates of kaolinite were observed. The average length of those stacks is around $6-8 \mu \mathrm{m}$, for a $4 \mu \mathrm{m}$ width. Some irregular pores may also be observed between those stacks whose radii are between 0.5 and $1.5 \mu \mathrm{m}$. Those inter-stack pores should correspond to the family of poorly graded bigger pores, whereas the family of well graded smaller pores should coincide with the intrusion of mercury inside stacks of platelets, in rather planar pores, which are unable to entrap it. The increase of stress would rearrange the stacks, and would involve to a similar extent the various sizes of this family of poorly graded inter-stack pores, without any shift towards lower porosity. The inter-platelet distance within the stacks should not then be changed. In fact, the remarkable superposition of the curves in the zone of smaller pores $(<0.15 \mu \mathrm{m})$, as well as the constitution of the stacks, as described by Tovey, would probably suggest a strong resistance to deformability of 
those stacks, owing to strong internal face to face links. In such a case, the validity of the deformable aggregates model has to be discussed, and the mechanism of consolidation may involve a rearrangement of undeformable stacks, which may be considered as rigid grains. Such a hypothesis should, however, be confirmed by a SEM observation.

\section{Authors' reply}

Nagaraj, Vatsala \& Srinivasa Murthy have demonstrated through examination of water contents that most of the water present in a soil is not participating in double layer interactions. This is in agreement with the comments made by Delage and the observations from mercury intrusion porosimetry as presented by the Authors in the Paper. Most of the pore space in the four soils examined was greater than $10 \mathrm{~nm}$ in radius.

The Authors agree with Nagaraj et al. in that mercury intrusion porosimetry may not be the right diagnostic test to prove or rule out the possibility of cluster formation with increase in stress. The degree of error at the lower levels of pore size approaches the same order of magnitude as the very small volumes measured. However, it is the fact that these volumes are so very small that is significant. Since most of the deformation occurs in very large pore spaces, the formation of clusters may not be important in the discussion of fabric changes due to consolidation.

Both Nagaraj et al. and Delage comment that a more accurate portrayal of the total effect of consolidation would be attained if the samples were not allowed to rebound before running mercury intrusion porosimetry. The Authors agree, however, practical difficulties have yet to be overcome with respect to finding a suitable technique which would preserve soil fabric while a sample is under load. This practical limitation also reduces the significance of clay cluster formation to consolidation. This form of deformation would be irreversible. Since rebound was allowed for the samples in this study, the deformation examined is limited to precisely the irreversible type. Therefore this present study more clearly demonstrates the relative importance of cluster formation to the amount of irreversible deformation than would a study on unrebounded samples.

The Authors agree that reversible deformation is of increasing importance at high stresses and for highly plastic soils, but have seen no evidence to support the contention by Delage that the pore space corresponding to reversible deformation (swelling porosity) will be entrapped.

Delage suggests that the slope of the mercury intrusion curve can be used to further demonstrate satisfactory results. Indeed, substantially
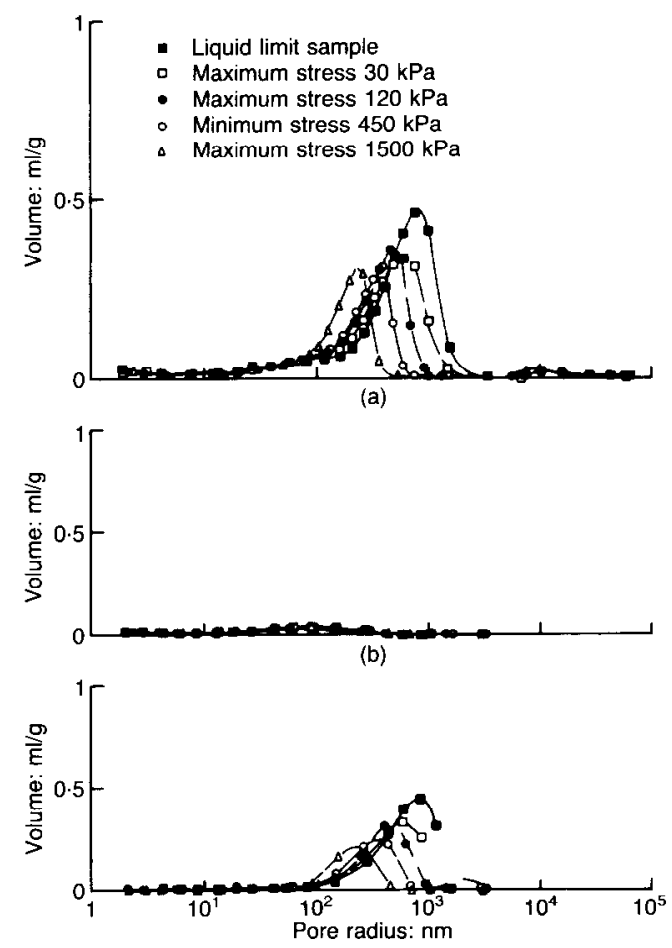

(c)

Fig. 13. Differential pore size distribution soil 1: (a) first intrusion; (b) second intrusion; (c) first minus second intrusion

more interpretation of fabric can be made by examining the slope of the cumulative volume intruded curves originally presented in the Paper. Fig. 13 is a plot of the differential distribution of soil 1 as determined by measuring the slope of the curves in Fig. 3. Consolidation deformation is readily apparent as a loss in differential peak height and as a shift towards smaller peak radii. Further examination and interpretation of the soils of this study using this method of data presentation is planned to be available in a comprehensive paper.

The comments of Delage regarding the mineralogy of the four soils is also further addressed in the forthcoming paper. Soils 1 and 2 which are different mineralogically (primarily quartz and kaolinite respectively) behave in a similar fashion. This can be explained, as noted by Delage, in that the kaolinite stacks of soil 2 are being rearranged by consolidation in a similar manner as are the silt grains of soil 1. The presence of montmorillonite connectors in soils 3 and 4 distinguishes both the initial fabric and the response to consolidation of these samples from that of soils 1 and 2 .

In summary, the Authors reiterate that mercury intrusion porosimetry is a very strong 
experimental technique for this type of fabric research. However, development of a procedure to preserve clay fabric while under stress is needed in order to proceed to examine the nature of the reversible component of consolidation behaviour.

\section{REFERENCES}

Delage, P. (1987). Microstructure and compressibility of some eastern Canadian sensitive soft clays. Proc. Int. Symp. on Geotechnical Engineering of Soft Soils, Mexico, 33-38.

Griffiths, F. J. \& Joshi, R. C. (1990). Clay fabric response to consolidation. Appl. Clay Sci. J. (in press).

Mitchell, J. K. (1976). Fundamentals of soil behaviour. New York: Wiley.

Nagaraj, T. S. \& Srinivasa Murthy, B. R. (1986). A critical reappraisal of compression index equations. Géotechnique 36, No. 1, 27-32.

Sridharan, A. \& Jayadeva, M. S. (1982). Double layer theory and compressibility of clays. Géotechnique 32, No. 2, 133-144.

Tovey, N. K. (1971). A selection of scanning electron micrographs of clays. CUED/C-SOIL S/TRSa (1971), University of Cambridge, Department of Engineering.

van Olphen, H. (1963). An introduction to clay colloid chemistry. Interscience Publishers, p. 41. 